\title{
Position Encoding Schemes for Linear Aggregation of Word Sequences
}

\author{
Diego Maupomé, Fanny Rancourt, Maxime D. Armstrong, Marie-Jean Meurs* \\ Université du Québec à Montréal, Montréal, QC, Canada
}

\begin{abstract}
Deep Averaging Networks (DANs) show strong performance in several key Natural Language Processing (NLP) tasks. However, their chief drawback is not accounting for the position of tokens when encoding sequences. We study how existing position encodings might be integrated into the DAN architecture. In addition, we propose a novel position encoding built specifically for DANs, which allows greater generalization capabilities to unseen lengths of sequences. This is demonstrated on decision tasks on binary sequences. Further, the resulting architecture is compared against unordered aggregation on sentiment analysis both with word- and character-level tokenization, to mixed results.
\end{abstract}

Keywords: Deep Averaging Network · Position Encoding · Neural Networks · Natural Language Processing · Sentiment Analysis

\section{Introduction}

Modeling natural language requires the decomposition of utterances into tokens, be they words or characters. However, language is sparse. Actually observed utterances make up an extremely small portion of the possible combinations of tokens. It is therefore apparent that in order to make a prediction on an entire phrase, the tokens that comprise it should be aggregated in a manner that is mindful of their role in the phrase. One key aspect of the roles of tokens is their order. Even in highly synthetic languages, the ordering of words can be used to convey information that the words themselves do not carry. As such, much work in Natural Language Processing (NLP) has been devoted to accounting for word order in modeling utterances. One such example is the decomposition of phrases into overlapping subsequences of words, called n-grams. These n-grams can then be treated in a structure-agnostic manner because they carry some sense of structure within them. They are however represented symbolically, so their semantic and structural sense is still acquired from surrounding n-grams. Moreover, the number of possible n-grams grows exponentially with respect to the length considered.

An alternative approach to the preservation of token order is to construct said order dynamically. That is, instead of attempting to consume the structure as a whole and then provide a summary of it, one might choose to construct that summary as the structure is being consumed in a predetermined order. Such is the case of Recurrent Neural Networks (RNN) [1] approaches, which read a phrase one word at a time and update their numerical state (see $[2,3]$ ). The final state would then represent a summary of the phrase relevant to the task. Given the non-linear nature of the function updating the state, the order of parsing of the tokens is embedded in this final state.

More recently, Transformer networks [4] have forgone recurrence altogether. The position of tokens is instead provided by a position encoding, a function mapping a natural number to a real vector, which is added to the symbol encoding (e.g. word embedding) of the token.

${ }^{*}$ meurs.marie-jean@uqam.ca

This article is (C) 2021 by author(s) as listed above. The article is licensed under a Creative Commons Attribution (CC BY 4.0) International license (https://creativecommons.org/licenses/by/4.0/legalcode), except where otherwise indicated with respect to particular material included in the article. The article should be attributed to the author(s) identified above. 
Thereafter, tokens are compared pairwise by the Self-Attention mechanism [5].

Transformers constitute the state of the art for many NLP benchmarks, often in the form of large models pre-trained on unannotated corpora by various schemes [6-8]. Nonetheless, such models can be computationally costly to train as well as detrimental to the environment [9]. Crucially, parameter count notwithstanding, the complexity of Transformers is quadratic with respect to the sequence length. While dilated variants aimed at longer documents have been proposed, [10], we are interested in reducing the computational cost at the core of the encoder. To this end, we take interest in Deep Averaging Networks (DANs) [11], which have achieved competitive results in text classification [12]. They are less computationally intensive than Transformers or RNNs as they operate on the average of the token representations. However, this aggregation does not account for token order, and as such, neither does the encoder. We therefore set out to investigate the following:

(1) Whether DANs can be amended to account for word positions in an efficient and generalizing manner

(2) Whether the resulting architecture exhibits benefits over DANs in natural language tasks

To this end, in Section 2 we study how positional encodings can be integrated into DANs. Section 3 measures how the resulting models fare in synthetic tasks centered on exploiting positional information. Further, a position encoding more apt at generalizing to unseen lengths of sequences is introduced. In Section 4, the resulting architecture is evaluated on natural language tasks. Finally, Section 5 concludes this paper.

\section{Integrating Existing Position Encodings}

Let $\boldsymbol{x}_{1}, \ldots, \boldsymbol{x}_{n}$ be a sequence of word vectors, forming a sentence, indexed by $i: 1 \leq i \leq n$. A DAN will compute the sentence representation as follows:

$$
\operatorname{dan}\left(\boldsymbol{x}_{1}, \ldots, \boldsymbol{x}_{n}\right)=\mathrm{ffn}\left(\frac{1}{n} \sum_{i=1}^{n} \boldsymbol{x}_{i}\right),
$$

where ffn denotes a feed-forward network. Thus, DANs average the representation of words making up a sentence and feed this representation through a deep feed-forward network. Our goal is to supplement the word representations to account for their position in the sequence. While Transformer encoders [4] integrate the position of a word to its symbol embedding by addition, this is not feasible for DANs. Indeed, if we were to simply add the position encoding to the symbol encoding, the linear nature of the average operation on which DANs are predicated would cause the sentence representation to lose track of which positions are assigned to which words. This holds for any position encoding.

We propose instead to integrate the position vector to the symbol vector in one of two ways, by an element-wise product, or a feed-forward network. Let $*$ denote the element-wise multiplication of vectors. The sentence representations computed by the proposed Positional DAN (p-dan) would be given by

$$
\operatorname{p}^{-\operatorname{dan}_{*}}\left(\boldsymbol{x}_{1}, \ldots, \boldsymbol{x}_{n}\right)=\text { ffn }\left(\frac{1}{n} \sum_{i=1}^{n} \boldsymbol{x}_{i} * \boldsymbol{p}(i)\right)
$$




\begin{tabular}{l|c|c} 
task & string & target \\
\hline \multirow{4}{*}{ precedence } & $1000 \underline{10}$ & 0 \\
& $1100 \underline{01}$ & 1 \\
& $0101 \underline{10}$ & 0 \\
& $010 \underline{10} \underline{1}$ & 1 \\
\hline \multirow{2}{*}{ palindrome } & 010010 & 1 \\
& 001001 & 0 \\
\hline \multirow{4}{*}{ parity } & 111001 & 0 \\
& 100010 & 1 \\
& 110011 & 1 \\
001111 & 0
\end{tabular}

Table 1. Examples of binary strings and their associated targets for each of the proposed decision tasks. For the precedence task, in this example, the model must decide whether the last 0 precedes the last 1.

in the product case, and by

$$
\text { p-dan }_{\mathrm{ffn}}\left(\boldsymbol{x}_{1}, \ldots, \boldsymbol{x}_{n}\right)=\mathrm{ffn}\left(\frac{1}{n} \sum_{i=1}^{n} \operatorname{ffn}\left(\boldsymbol{x}_{i}+\boldsymbol{p}(i)\right)\right)
$$

in the feed-forward network case. While the feed-forward approach is more expressive than the product one, it also has the disadvantage of adding to the parameter count and computational burden of the model. We conduct experiments on synthetic data to evaluate the capacity of these approaches to discern the position of tokens in a sequence.

\section{Experiments on Synthetic Data}

We propose three different decision tasks on strings of characters aimed at establishing the capacity to distinguish the positions of characters in a string of different position encodings and integrations in a p-dan. The first task requires two distinguished characters and asks to decide whether the last instance of one of these characters arrives before the last instance of the other. Note that both of these characters can occur multiple times in the string. We refer to this task as the precedence task. The second task requires simply to decide whether the string is a palindrome, and we refer to it as the palindrome task. The final task is more complex and also requires two distinguished characters. The model is asked to decide whether the last sequence of consecutive occurrences of one of these characters uninterrupted by the other is of count equal to the converse. We refer to this task as the parity task. Table 1 presents some examples of strings and their associated targets for each task.

To enhance the difficulty of the tasks and increase the proportion of eligible strings, we use an alphabet of only two characters. Indeed, sequences containing several occurrences of a few possible characters will require the model to separate these occurrences by position without the possibility of relying on simple associations between characters and positions. For each task, we generate 50k strings of length 32 , randomly selecting $10 \mathrm{k}$ for validation, in a stratified manner. The strings generated are not unique. Therefore, we remove any strings present in the training set from the validation set. Strings are generated in equal proportions for both classes. In the case of the parity task, the two possible negative cases (one of the two distinguished symbols occurring more or viceversa) are also generated in roughly equal proportions. Furthermore, in the case of the palindrome task, all non-palindrome strings are the repetition of a substring to prevent the models from relying on the parity of character counts to approximate an answer. Finally, in order to isolate the effects of different position encodings and integrations, the symbol embedding vectors, $\boldsymbol{x}_{i}$, remain fixed for these 


\begin{tabular}{|c|c||c|c|c|}
\hline \multicolumn{2}{|c||}{} & \multicolumn{3}{|c|}{ Task } \\
\hline Encoding & Integration & precedence & palindrome & parity \\
\hline \hline \multirow{3}{*}{ Sinusoidal } & $\boldsymbol{x}_{i}$ & 57.5 & 50.2 & 51.83 \\
& $\boldsymbol{x}_{i}+\boldsymbol{p}(i)$ & 57.2 & 50.3 & 50.0 \\
& ffn $\left(\boldsymbol{x}_{i}+\boldsymbol{p}(i)\right)$ & 100.0 & 98.6 & 50.0 \\
& $\boldsymbol{x}_{i} * \boldsymbol{p}(i)$ & 100.0 & 100.0 & 50.0 \\
\hline \multirow{3}{*}{ Embedding } & $\boldsymbol{x}_{i}$ & 57.6 & 50.6 & 52.4 \\
& $\boldsymbol{x}_{i}+\boldsymbol{p}(i)$ & 57.4 & 50.2 & 50.0 \\
& ffn $\left(\boldsymbol{x}_{i}+\boldsymbol{p}(i)\right)$ & 100.0 & 50.1 & 50.0 \\
& $\boldsymbol{x}_{i} * \boldsymbol{p}(i)$ & 98.7 & 99.8 & 68.0 \\
\hline
\end{tabular}

Table 2. Validation accuracy (\%) on the three synthetic tasks for the proposed integrations of learned position embedding vectors and sinusoidal position encoding. Random performance is $50 \%$ for all tasks.

experiments. They are random, dense, orthogonal vectors.

We begin with experiments comparing the integration of position encodings with each of two position encodings: sinusoidal position encodings [4] and learned position embeddings [13]. The dimension of the input space is set to 64. Further, all models have three dense layers of 64 units processing the aggregated string. This yields models with $12 \mathrm{k}$ parameters for $\mathrm{p}-\mathrm{dan}_{*}$ and $25 \mathrm{k}$ for $\mathrm{p}$-dan $\mathrm{ffn}_{\mathrm{fn}}$. Each layer uses ReLU activation [14]. The models are trained by the Adam optimizer [15] over 30 epochs, and we report the best results obtained in validation. We compare both of the proposed integration approaches to simple additive integration and no positional information, fully expecting these to fail all three tasks. The results are presented in Table 2. As expected, only the proposed positional integrations succeed at the precedence task. The above-random performance of models without positional information is likely due to their classifying strings with a higher count of 1 s as positive. On the other hand, only the multiplicative approach succeeds at the palindrome task for both position encodings. All models perform poorly at the parity task.

These first results notwithstanding, a more important aspect of these encodings and integrations is whether they successfully generalize to previously unseen lengths. This is unlikely for position embedding vectors, for example. These are "named" vectors with no parameter sharing. Therefore, there is, a priori, no mechanism in place that would allow to generalize to previously unseen lengths. To this end, we introduce a new position encoding in the following section.

\subsection{Normalized Relative Distance}

Recently, relative position encodings for Transformers [10] have increased in popularity. This is in line with the pairwise contextualization effected by the self-attention mechanism that Transformers are predicated on: each word need not be aware of the absolute position of the word it is matched with, only the relative position. However, so far, our approach has been better fit to global positional information, as the aggregation over the sequence happens at once, without any pairwise transformation. Nonetheless, we describe how, by reasoning in terms of relative positions, we can obtain a global position encoding suited for our approach.

Suppose we wished to supplement the word or token representations with those of neighbouring words. Further, we wish for this amended representation to account for the relative position of neighbours. Given a relative position encoding, $r: \mathbb{Z} \longrightarrow \mathbb{R}^{d}$, the representation 
of each word, $\boldsymbol{x}_{i}$, is replaced by a softmax-weighted sum of the sequence:

$$
\boldsymbol{x}_{i} \leftarrow \sum_{j=1}^{n} \frac{e^{\boldsymbol{r}(j-i)}}{\sum_{k=1}^{n} e^{\boldsymbol{r}(k-i)}} * \boldsymbol{x}_{j}
$$

Here, exponentiation is applied element-wise. When averaging over an entire sequence $\boldsymbol{x}_{1}, \ldots, \boldsymbol{x}_{n}$, we obtain:

$$
M=\frac{1}{n} \sum_{i=1}^{n} \sum_{j=1}^{n} \frac{e^{\boldsymbol{r}(j-i)}}{\sum_{k=1}^{n} e^{\boldsymbol{r}(k-i)}} * \boldsymbol{x}_{j}
$$

In doing so, the sum can be rearranged in the following manner:

$$
M=\frac{1}{n} \sum_{i=1}^{n}\left(\boldsymbol{x}_{i} * \sum_{j=1}^{n} \frac{e^{\boldsymbol{r}(i-j)}}{\sum_{k=1}^{n} e^{\boldsymbol{r}(k-j)}}\right)
$$

In other words, the order of summation can be swapped so that each token is weighted by the sum total of the influence the token has on every other token. This is illustrated in Figure 1.

We set $\boldsymbol{r}$ as $\boldsymbol{r}(d)=d \boldsymbol{w}$, where $\boldsymbol{w} \in \mathbb{R}^{d}$ is a vector of learned parameters. Thereby, given that softmax normalization is translation invariant, the position of the observing token (i.e. the one replaced in Equation 3.1) is irrelevant. That is, it is implied by the positions of neighbors. Thus, Equation 3.2 can be reduced to the encoder we propose, Normalized Relative Distance (NRD) p-dan:

$$
\mathrm{p}-\operatorname{dan}_{n r d}\left(\boldsymbol{x}_{1}, \ldots, \boldsymbol{x}_{n} ; \boldsymbol{w}\right)=\sum_{i=1}^{n} \frac{e^{i \boldsymbol{w}}}{\sum_{k=1}^{n} e^{k \boldsymbol{w}}} * \boldsymbol{x}_{i}
$$

Negative components of $\boldsymbol{w}$ will favor the left-hand side of a sequence, and positive ones will favor the right-hand side. Larger absolute values will favor further positions. We initialize $\boldsymbol{w}$ by sampling from a zero-centered truncated normal distribution.

Like that of the other p-dan encoders studied earlier, the complexity of NRD is linear in the length of the sequence. Contrary to sinusoidal position encodings, a shift in position cannot be expressed as a linear map. However, this is consistent with our initial construction of the modified word representations, where a shifted word would need to know what words caused the shift and how close they are. Moreover, this position encoding requires not only to know the position of a word with respect to the start of the sequence but also with respect to its end. This makes intuitive sense, as the role of a word in a sentence is easier to establish if the distance to both beginning and end of said sentence is known.

We proceed with experiments on the previously described synthetic tasks. With the same experimental settings described earlier, this new approach achieves accuracies of $100 \%$, $99.53 \%$ and $97.6 \%$ on the precedence, palindrome and parity tasks. Further, in order to test the generalization capabilities of this approach and the ones studied earlier, we carry out experiments varying the lengths of the sequences considered. These experiments compare NRD to p-dan ${ }_{*}$ with sinusoidal position encoding as well as position embeddings. In one set of experiments, the models are trained on sequences of lengths varying from 16 to 32 and validated on sequences of lengths from 33 to 48 . These experiments are then repeated with the ranges of sequence lengths reversed, thus assessing the capabilities of the models of transposing the task to longer and shorter sequences. All other settings are identical to those described earlier. The results, presented in Table 3, show that for these synthetic tasks, NRD are far more apt at generalizing to unseen lengths of sequences. 


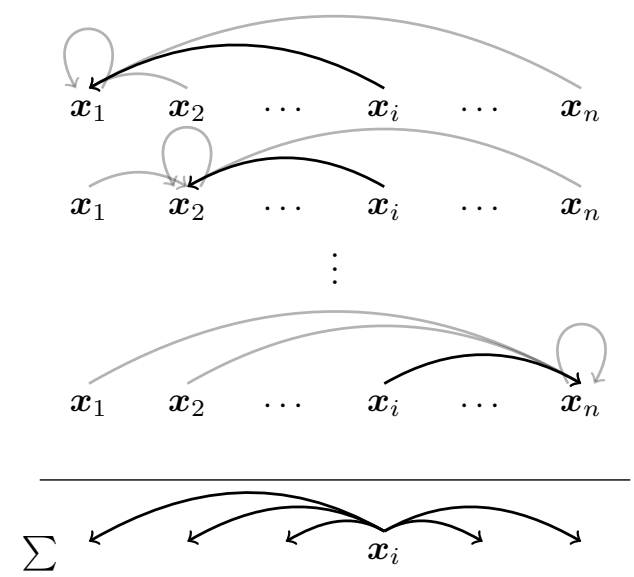

Figure 1. The proposed position encoding weighs each token as a cross-normalized sum of its contributions to other tokens.

\begin{tabular}{|c||c|c|c|c|c|c|}
\hline \multicolumn{1}{|c||}{} & \multicolumn{6}{c|}{ Task } \\
\hline Encoding & \multicolumn{2}{|c|}{ precedence } & \multicolumn{2}{c|}{ palindrome } & \multicolumn{2}{c|}{ parity } \\
\hline \hline Sinusoidal & 52.1 & 50.1 & 49.0 & 51.0 & 51.3 & 51.2 \\
\hline Embedding & 50.3 & 50.8 & 50.3 & 50.5 & 50.4 & 50.1 \\
\hline NRD & 100.0 & 100.0 & 82.9 & 86.4 & 96.6 & 95.9 \\
\hline
\end{tabular}

Table 3. Validation accuracy (\%) on the three synthetic tasks when generalizing to unseen lengths (longer |shorter). Positions are integrated by element-wise multiplication $\left(\mathrm{p}-\mathrm{dan}_{*}\right)$ for sinusoidal position encoding and position embeddings. Random performance is $50 \%$ for all tasks.

However, while these experiments show the capability of the proposed approach to differentiate the positions of tokens and separate subsequences, it is our main goal to test the pertinence of these capabilities on natural language data. The experiments carried out in NLP are presented in the following section.

\section{Natural Language Experiments}

Following Iyyer, Manjunatha, Boyd-Graber, and Daumé III [11], the proposed approach is evaluated on three sentiment analysis datasets. All three datasets consist of film reviews. The Rotten Tomatoes dataset (RT) [16] contains 11k sentences from film reviews classified as either positive or negative in equal proportions. The larger IMDb dataset (IMDB) [17] contains a total of 50k reviews divided evenly between positive and negative ones. Finally, while the Stanford Sentiment Treebank (SST) [18], made up of 11k reviews, offers a finergrained syntax-tree sentiment annotation, we take only the label associated with the whole sentence. For all datasets, given the equal proportions between the negative and positive classes, it is customary to report the binary classification accuracy.

\subsection{Masked Language Model Pretraining}

In order to enhance the performance of neural models on natural language data, it is common practice to pre-train them on some unsupervised task. We employ Masked Language Modeling (MLM) with dynamic masking [7]. MLM consists in obscuring tokens in a sequence and asking the model to produce those hidden tokens. As such, it is not a proper 


\begin{tabular}{|l|l|c||c|c|c|}
\hline Tokenization level & Model & Size & RT & SST & IMDB \\
\hline \hline \multirow{3}{*}{ Word } & dan & \multirow{3}{*}{$13.3 \mathrm{M}$} & 76.7 & 77.1 & 88.1 \\
& p-dan $_{n r d}$ & & 77.0 & 76.8 & 88.1 \\
& pre-trained p-dan $_{n r d}$ & & 77.2 & 87.9 \\
\hline \multirow{3}{*}{ Character } & dan & & 50.0 & 59.1 & 50.0 \\
& p-dan & & \\
& pretrained p-dan $_{n r d}$ & & 74.1 & 73.0 & 80.0 \\
\hline \multirow{2}{*}{ Word } & dan[11] & - & 77.3 & 83.2 & 88.8 \\
& dan[12] & - & 72.2 & 77.5 & - \\
\hline
\end{tabular}

Table 4. Test set accuracy (\%) and parameter counts on the three sentiment analysis tasks for our model and related approaches (where available).

language model (a probability distribution over sequences of words) as it is a discriminative model, unlike recurrent language modeling [19]. Nonetheless, MLM, in different forms, has enjoyed much success as a pre-training scheme for Transformers. Given the large size of the output space (the size of the vocabulary) normalizing the loss function for each training instance can be computationally expensive. Instead, one can use negative sampling methods, i.e. comparing the true obscured token against only a sample of negative candidates, rather than the entire vocabulary. We employ a variant of noise contrastive estimation amounting to ranking the true token above the noise tokens [20]. In the discriminative case, this requires weaker assumptions about the nature of the model than matching the true candidate against each negative one [21].

We perform MLM on the WikiText-103 dataset [22], comprising selected Wikipedia articles totalling over 103 million words. We restrict the original vocabulary of $268 \mathrm{k}$ words to the $50 \mathrm{k}$ most frequent ones. The training procedure is as follows. For each training step, we create a batch by sampling 16 documents, from each of which 8 sequences of 128 words are sampled. Following [7], these sequences are formed by taking a sample of contiguous sentences to fill the set length. The 1024 noise candidates are sampled from the $20 \mathrm{k}$ most frequent words (the 100 most frequent excluded) according to their frequency. For efficiency, this sample is taken preemptively and is shared across sequences in a training step. At each step, 16 tokens in each sequence are masked at random. The models are trained by the Adam optimizer [14], applying early stopping as per the validation loss.

\subsection{Sentiment Analysis}

We compare the proposed approach to DANs. All models operate on random word embeddings of size 256. The feed-forward network consists of 7 layers with ELU activation [23]. The models are trained by the Adam optimizer by batches of 64 observations over 20 epochs.

Initial results in classification show little difference between p-dan and DAN models. pdan models overfit the training set quite heavily, requiring aggressive regularization in the form of dropout to improve performance on the validation sets. This is also the case for pre-trained models. In order to address this issue and exploit positional information, we extend the approach to the character level. That is, each word is treated as a sequence of characters encoded separately by a word encoder. These encoded words are then treated as regular word vectors by a sentence encoder. By descending to the character level, the input space decreases dramatically in size and so do the parameter count of models, decreasing the chance of overfitting. In MLM pre-training, the premise remains the same, only candidate words are sequences of characters encoded by the same word encoder used for the sentence. Our expectations for these experiments were a clearer difference in performance between 
DAN and p-dan models. Moreover, we expected pretraining to have a greater impact on classification performance as models will learn deeper word representations.

The results for all models and the associated parameter counts are presented in Table 4, in addition to results reported in previous work. Our results are consistent with those described by Iyyer, Manjunatha, Boyd-Graber, and Daumé III [11]. However, our word-level DAN does not achieve the results reported on the SST dataset but is closer to those reported by Cer, Yang, Kong, Hua, Limtiaco, St. John, Constant, Guajardo-Cespedes, Yuan, Tar, Strope, and Kurzweil [12]. This difference might be due to the fact that our experiments do not use word dropout. As previously mentioned, word-level models achieve comparable performance, whether they include positional information or not. This is also consistent with the conclusions drawn by Iyyer, Manjunatha, Boyd-Graber, and Daumé III [11], where a DAN achieve comparable performance to a recursive neural network.

As for character-level models, while a drop in performance from DANs was to be expected, interestingly, they perform on par with random labeling on the RT and IMDB datasets. Further, while there is a greater benefit to pre-training for these models, the performance still falls short of that achieved by word-level models. Nonetheless, given the small size of the models, the results obtained are interesting. Given the overfitting we observed from p-dan at the word level, it seems p-dan are much more parameter-efficient than regular DAN.

\section{Conclusion}

The work presented has studied the integration of position information in averaging sequence encoders. Such encoders have the advantage of computational complexity that is linear in the length of the sequences. Moreover, a position encoding constructed with such encoders in mind has been introduced. This encoder shows greater generalization capabilities than the other approaches considered on synthetic data specifically conceived to evaluate the discernment of positions. On sentiment analysis, the proposed approaches do not seem to offer any advantages over unordered aggregation, when tokenizing text at the word level. This is consistent with the literature. At the character-level, given the small size of our models, DAN failed to progress beyond random performance, contrary to positional models. Nonetheless, while working at the character level dramatically decreases the parameter count, it of course increases the length of sequences. As such, future work could involve tokenization between the word and character levels, such as byte-pair encodings [24]. Further, a more detailed comparison in terms of results and computational burden with Transformers and the recently proposed Performer [25], which offers complexity linear with respect to sequence length by approximating full self-attention, is needed. More importantly, future work in NLP could focus on tasks where word order might be more decisive, such as natural language inference or linguistic acceptability.

Reproducibility: The source code of the proposed systems is licensed under the GNU GPLv3.

\section{Acknowledgements}

This research was enabled in part by support provided by Calcul Québec and Compute Canada. MJM acknowledges the support of the Natural Sciences and Engineering Research Council of Canada [NSERC Grant number 06487-2017] and the Government of Canada's New Frontiers in Research Fund (NFRF), [NFRFE-2018-00484]. 


\section{References}

[1] M. Jordan. "Attractor Dynamics and Parallelism in a Connectionist Sequential Machine". In: Proceedings of the Eighth Annual Conference of the Cognitive Science Society (Erlbaum, Hillsdale, NJ), 1986. 1986.

[2] Z. Yang, Z. Dai, R. Salakhutdinov, and W. W. Cohen. "Breaking the Softmax Bottleneck: A High-Rank RNN Language Model". In: arXiv preprint 1711.03953 (2018).

[3] D. Maupomé and M.-J. Meurs. "Language Modeling with a General Second-Order RNN". In: Proceedings of the 12th Language Resources and Evaluation Conference.

[4] A. Vaswani, N. Shazeer, N. Parmar, J. Uszkoreit, L. Jones, A. N. Gomez, Ł. Kaiser, and I. Polosukhin. "Attention is All You Need". In: Advances in neural information processing systems.

[5] J. Cheng, L. Dong, and M. Lapata. "Long Short-Term Memory-Networks for Machine Reading". In: arXiv preprint 1601.06733 ().

[6] J. Devlin, M.-W. Chang, K. Lee, and K. Toutanova. "BERT: Pre-training of Deep Bidirectional Transformers for Language Understanding". In: arXiv preprint 1810.04805 (2019).

[7] Y. Liu, M. Ott, N. Goyal, J. Du, M. Joshi, D. Chen, O. Levy, M. Lewis, L. Zettlemoyer, and V. Stoyanov. "RoBERTa: A Robustly Optimized BERT Pretraining Approach". In: arXiv preprint 190\%.11692 ().

[8] K. Clark, M.-T. Luong, Q. V. Le, and C. D. Manning. "ELECTRA: Pre-training Text Encoders as Discriminators Rather Than Generators". In: arXiv preprint 2003.10555 (2020).

[9] R. Schwartz, J. Dodge, N. A. Smith, and O. Etzioni. "Green ai". In: Communications of the ACM 63.12 (2020), pp. 54-63.

[10] Z. Dai, Z. Yang, Y. Yang, J. Carbonell, Q. V. Le, and R. Salakhutdinov. "Transformer-XL: Attentive Language Models Beyond a Fixed-Length Context". In: arXiv preprint 1901.02860 (2019).

[11] M. Iyyer, V. Manjunatha, J. Boyd-Graber, and H. Daumé III. "Deep Unordered Composition Rivals Syntactic Methods for Text Classification". In: Proceedings of the 53rd Annual Meeting of the Association for Computational Linguistics and the 7th International Joint Conference on Natural Language Processing.

[12] D. Cer, Y. Yang, S.-y. Kong, N. Hua, N. Limtiaco, R. St. John, N. Constant, M. GuajardoCespedes, S. Yuan, C. Tar, B. Strope, and R. Kurzweil. "Universal Sentence Encoder for English". In: Proceedings of the 2018 Conference on Empirical Methods in Natural Language Processing: System Demonstrations.

[13] J. Gehring, M. Auli, D. Grangier, D. Yarats, and Y. N. Dauphin. "Convolutional Sequence to Sequence Learning". In: Proceedings of the 34th International Conference on Machine Learning-Volume 70.

[14] R. H. Hahnloser and H. S. Seung. "Permitted and Forbidden Sets in Symmetric ThresholdLinear Networks". In: Advances in neural information processing systems.

[15] D. P. Kingma and J. Ba. "Adam: A Method for Stochastic Optimization". In: arXiv preprint 1412.6980() .

[16] B. Pang and L. Lee. "Seeing Stars: Exploiting Class Relationships for Sentiment Categorization with Respect to Rating Scales". In: Proceedings of the 43rd annual meeting on association for computational linguistics.

[17] A. Maas, R. E. Daly, P. T. Pham, D. Huang, A. Y. Ng, and C. Potts. "Learning Word Vectors for Sentiment Analysis". In: Proceedings of the 49th annual meeting of the association for computational linguistics.

[18] R. Socher, A. Perelygin, J. Wu, J. Chuang, C. Manning, A. Ng, and C. Potts. "Parsing With Compositional Vector Grammars". In: Proceedings of the 51st Annual Meeting of the Association for Computational Linguistics. 2013.

[19] T. Mikolov, M. Karafiát, L. Burget, J. Černockỳ, and S. Khudanpur. "Recurrent Neural Network based Language Model". In: Eleventh annual conference of the international speech communication association. 2010.

[20] R. Jozefowicz, O. Vinyals, M. Schuster, N. Shazeer, and Y. Wu. Exploring the Limits of Language Modeling. 2016. 
[21] Z. Ma and M. Collins. "Noise Contrastive Estimation and Negative Sampling for Conditional Models: Consistency and Statistical Efficiency". In: Proceedings of the 2018 Conference on Empirical Methods in Natural Language Processing.

[22] S. Merity, C. Xiong, J. Bradbury, and R. Socher. "Pointer Sentinel Mixture Models". In: arXiv preprint 1609.07843 (2016).

[23] D.-A. Clevert, T. Unterthiner, and S. Hochreiter. Fast and Accurate Deep Network Learning by Exponential Linear Units (ELUs). 2016.

[24] R. Sennrich, B. Haddow, and A. Birch. Neural Machine Translation of Rare Words with Subword Units. 2016.

[25] K. Choromanski, V. Likhosherstov, D. Dohan, X. Song, A. Gane, T. Sarlos, P. Hawkins, J. Davis, A. Mohiuddin, L. Kaiser, D. Belanger, L. Colwell, and A. Weller. "Rethinking Attention with Performers". In: arXiv preprint 2009.14794 (2021). 